\title{
A Cluster-based Routing Protocol and FAULT DETECTION FOR WIRELESS SENSOR NETWORK
}

\author{
Slaheddine Chelbi ${ }^{1}$ and Riadh Moussi ${ }^{2,3}$ \\ ${ }^{1}$ Department of computer sciences, College of Sciences and Arts in Unaizah, Qassim \\ University, Unaizah, Kingdom of Saudi Arabia \\ ${ }^{2}$ Faculty of science and art al-ula. Taibah University \\ ${ }^{3}$ Research Unit of Valuation and Optimization of Resource, Faculty of Science and \\ Technology of Sidi Bouzid, University of Kairouan, Tunisia
}

\begin{abstract}
In Wireless Sensors Networks (WSN) based application, a large number of sensor devices must be deployed. Energy efficiency and network lifetime are the two most challenging issues in WSN. As a consequence, the main goal is to reduce the overall energy consumption using clustering protocols which have to ensure reliability and connectivity in large-scale WSN. This work presents a new clustering and routing algorithm based on the properties of the sensor networks. The main goal of this work is to extend the network lifetime via charge equilibration in the WSN. According to many errors with sensing devices and to have greater data accuracy, we use a quorum mechanism. The proposed algorithms are evaluated widely and the results are compared with related works. The experimental results show that the proposed algorithm provides an effective improvement in terms of energy consumption, data accuracy and network lifetime.
\end{abstract}

\section{KEYWORDS}

Wireless sensor network, clustering, routing protocol, load balancing, data accuracy

\section{INTRODUCTION}

A Wireless Sensor Networks (WSN) contains groups of interconnected devices with energyconstrained, low processing power and limited wireless communication capabilities [1]. These devices communicate and collaborate to accomplish a given task.

Due to the low cost and facility of deployment of the sensor node, WSN has a wide range of applications in different fields, such as industries, health care, environment, agricultures, surveillance, military, etc. [2] [3] [4][5][6].

Since sensor nodes are usually driven by limited and irreplaceable power sources, improving the energy consumption efficiency in a WSN is therefore a crucial issue. That's way, using energyaware algorithms is greatly significant [7] [8] [9]. To prolong the network lifetime, data aggregation has been used to remove the data redundancy and to reduce the communication load [10] [11] [12] [13].

In each cluster, an elected node, called Cluster Head $(\mathrm{CH})$, collects these data and forwards them to the Base Station (BS) after possibly having performed appropriate data aggregation. As mentioned before, the aim of aggregation is to extend the lifetime of the network by reducing resource consumption. The role of the cluster head turns around the nodes to ensure distribution of the load between nodes [14] [15] [16]. In a large-scale WSN, many researchers have proved that multi-hop inter-cluster communication mode is typically more energy efficient on account of the features of the wireless channel [17][18]. Thus, it is better to let $\mathrm{CH}$ collaborate to 
transmit their data to the BS. Nevertheless, $\mathrm{CH}$ carries some extra workload contributed by their cluster members as they receive the sensed data from their cluster members, aggregate them and communicate it to the BS. In addition, when $\mathrm{CH}$ works together to transfer their data to the BS, the $\mathrm{CH}$ nearer to the BS are overloaded and tend to die much faster. Consequently, the sensing area becomes uncovered and the network turns partitioned.

To solve this major problem, the proposed protocol chooses a collection of powerful relay nodes from the usual sensor nodes in order to reduce the energy consumption of $\mathrm{CH}$ and avoid premature death of relay $\mathrm{CH}$ close to the BS.

In addition, WSN is prone to many failures, which can be classified in three types: hardware failures, software failures and communication failures. Faults can be categorized according to data: offset fault, gain fault, stuck-at fault, out of bounds, spike faults and data loss fault [19]. All the factors will reduce the accuracy of collected data. Therefore, the fault detection plays a crucial role due to sensor resources limitation. Moreover, the fault detection avoids negative alerts, improves the accuracy of monitoring data and reduces the overall energy consumption.

In this work, we propose a fully distributed energy-efficient cluster-based algorithm for WSN. Our protocol aims to guarantee the distribution of load between nodes. In order to achieve this goal, we will divide the nodes into two groups: sensing and relaying nodes. The relaying nodes act like gateways and are responsible to relay the collected data within multiple hops to the BS.

The rest of this paper is structured as follows: Section 2 presents some related works. Section 3 introduces our proposed protocol; Section 4 shows the analysis and experiment results. Finally, section 5 draws the conclusion.

\section{RELATED WORK}

A WSN has attracted the attention of researchers in several difficult areas in the last few years. Energy conservation is the most important challenge in those networks. The design of clustering and routing process is an important part to conserve energy in WSN and to prolong the network lifetime. Some clustering algorithms have been proposed in the literature to distribute load among nodes and to solve the energy constraint problem.

A cluster-based routing protocol with non-uniform node distribution (EADC) has been introduced [20]. EADC is a competition-based algorithm, where CHs are chosen on the basis of the ratio between its residual energy to neighbors' average residual energy. Each $\mathrm{CH}$ selects the node with higher energy and less cluster members as relay to forward data. The CH's forwarding role is increased to address the imbalanced energy consumption induced by nonuniform node distribution.. The protocol prolongs the network lifetime. EADC can achieve a good cluster heads distribution and balances the energy dissipation among nodes. The major problem is that this technique does not assure connectivity among the $\mathrm{CH}$.

In [21], an Event Clustering Routing Protocol based on Consensus (ECRPC) is proposed for WSN. The key concept behind ECRPC is that no message will be sent to the Base Station (BS) until a consensus has been reached. In order to select the next relay, a tradeoff is made between residual energy and link cost. In some situations, the priority is given to length relay at the expense of consumed energy or vice versa.

An unequal clustering and multi-hop routing algorithm to extend the network lifetime has been proposed [22]. In this protocol, $\mathrm{CH}$ is chosen on the basis of local information i.e., the residual energy of neighboring nodes. To alleviate the hot spot problem, nodes are grouped into clusters of unequal sizes. As a result, $\mathrm{CH}$ nearer to the BS is predicted to have fewer cluster members. Therefore, less energy will be consumed during the intra-cluster communication and more energy recourse can be maintained for the inter-cluster relay traffic. However this idea is not always efficient for networks with non-uniform node distribution.

In [23], an optimal cluster head selection based on particle swarm optimization (PSO-ECHS) has been introduced [23]. This approach was based on an effective scheme of particle encoding and objective function. The $\mathrm{CH}$ is chosen based on three criteria: intra-cluster communication, 
distance to BS and residual energy of sensor nodes. In addition, each normal sensor nodes select its cluster head based on the derived weight function.

In [17], an effective particle swarm optimization (PSO) based on clustering and routing algorithms for WSN has been proposed. The main goal of this protocol is to minimize the energy consumption in order to improve the network lifetime. The routing algorithm has been established by considering a trade-off between transmission distance and the number of hopcount. In the data transmission process, the routing overhead of the $\mathrm{CH}$ is taken into consideration to equilibrate the energy consumption of the $\mathrm{CH}$.

In [18], a decentralized cluster-based routing algorithm is proposed. In this approach, local information such as residual power, distance to the BS and proximity to its neighbors are used to choose $\mathrm{CH}$ nodes. To construct a routing tree, the proposed protocol selects a next hop relay node with more residual energy, in a low-density area.

An unequal clustering based on Fuzzy logic-based and a routing protocol based on an Ant Colony Optimization (ACO) based has also been proposed [13]. The main goal of this approach is to solve the hot spot problem and prolong the network lifetime. First, Fuzzy logic selects $\mathrm{CH}$. Next, unequal clusters are formed based on residual energy, node degree and distance to BS, its neighbors, and center of cluster. ACO-based routing technique was used to establish an efficiently and reliably route from $\mathrm{CHs}$ to BS.

The main goal of the aforementioned algorithms is to use the clustering technique to minimize and balance the energy consumption of nodes. The main advantages of clustering are:

- facilitates the data aggregation, hence the number of unnecessary data transmissions will be reduced and the overall energy will be conserved,

- simplifies the data routing process,

- retains communication bandwidth. However, the major problem is the $\mathrm{CH}^{\prime}$ significant burden, resulting in fast death of nodes.

\section{THE PROPOSED PROTOCOL}

In this section, the proposed Advanced Energy Efficient Clustering (AEEC) mechanism will be presented in detail. We suppose that the WSN consists of a BS and a set of homogeneous sensor nodes. These devices are randomly deployed over a sensing field.

To simplify the network model, we adopt the following hypothesis:

- All nodes have power control to alter the amount of transmission power.

- BS doesn't have any energy constraints.

- Sensor nodes are energy constrained

- Each node has a unique identity.

- All sensors are GPS-enabled

Our primary objective in a cluster-based routing algorithm is to minimize the cost of transmitting messages by building a routing tree that covers the entire network. Since CHs closer to the BS relay too much data from all over the network, it may lead to the premature death of those $\mathrm{CH}$. To avoid this problem, the proposed protocol selects a collection of powerful relay nodes from the normal sensor nodes and a routing tree would be constructed on elected relay nodes set to forward data to the BS.

AEEC is a distributed cluster-based algorithm. The selection of relay nodes or cluster heads is mainly based on the residual energy.

Let $r$ represents a specific round and let $S(r), R(r)$ and $C H(r)$ denote the set of sensing nodes, the set of nodes selected as relaying nodes and the set of nodes selected as cluster head at the round $r$ respectively. After a given number $(\mathrm{Nb})$ of iterations, the current round is finished, and another round is began.

At each round, the sensing nodes $S(r)$ are responsible for sensing data, the cluster heads $C H(r)$ are responsible for collecting the sensed data generated by the nodes of $S(r)$ and the relaying 
nodes $R(r)$ are responsible for relaying the data toward the BS along the best transmission path. Relay nodes would be selected in such a way that the constructed route to the BS becomes optimal. To pick relay and $\mathrm{CH}$ nodes, we use local information of each node, such as residual energy and distance to the BS. Residual energy is an important factor to avoid the premature death of nodes and to distribute load among nodes.

$$
\operatorname{State}\left(s_{i}\right)=\left\{\begin{array}{c}
\text { "Relay node" } \\
\text { "Cluster Head" } \\
\text { "Member" }
\end{array}\right.
$$

As shown in Figure 1, the whole process is performed in four phases: Relay nodes election, $\mathrm{CH}$ election and clustering, routing phase and transmission phase.

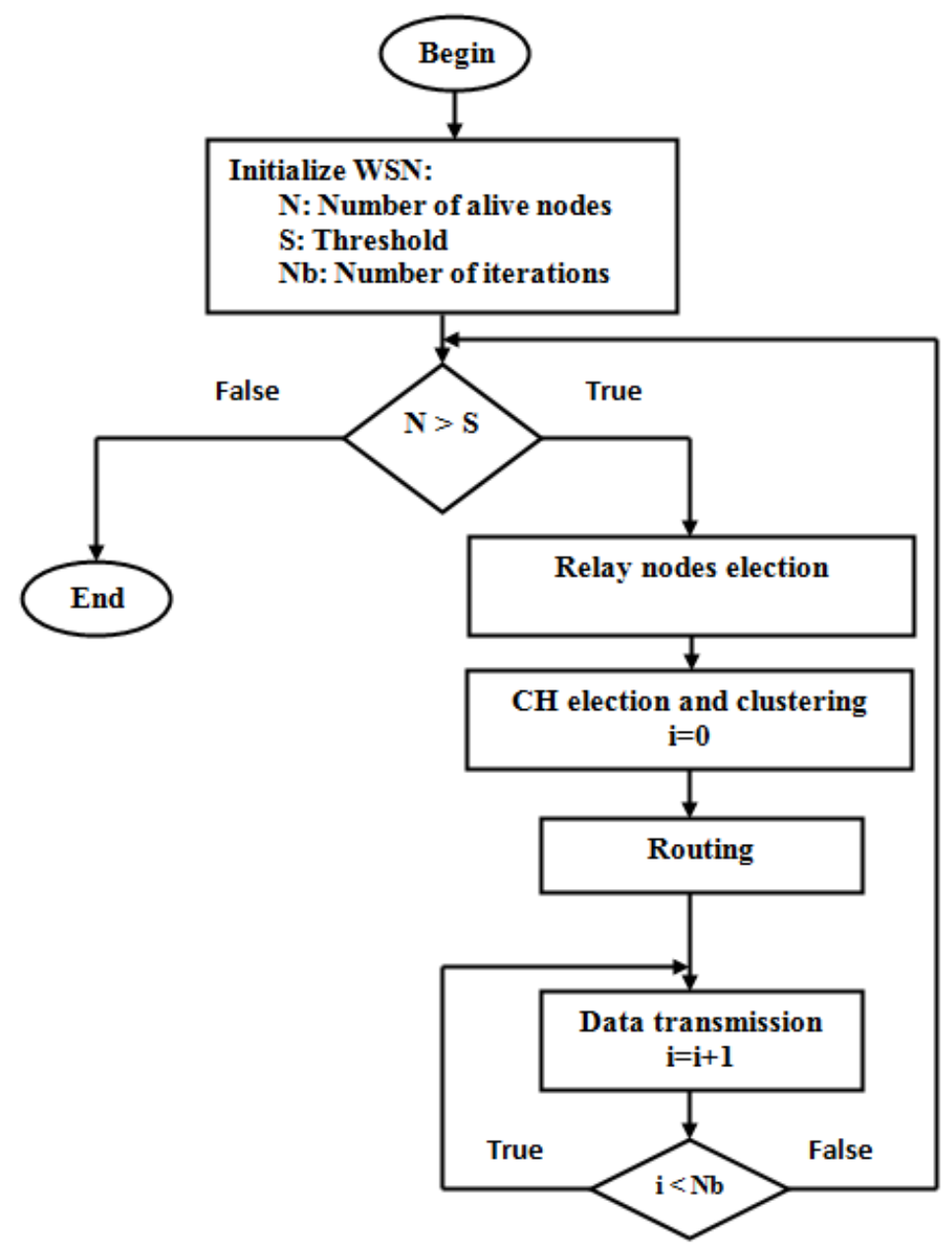

Figure 1 An overview of the proposed protocol

\subsection{Relay node election phase}

In this phase, relay nodes are selected among sensor nodes and concurrently, routing tree will be constructed among elected relay nodes. AEEC is a distributed and competitive algorithm. The election of tentative relay nodes is essentially based on residual energy. The role of relay node is periodically rotated among the nodes to distribute the energy consumption among nodes. The relaying nodes act like gateways and are responsible to relay the collected data via multiple hops to the BS. 
The whole network is virtually divided into rings, and the width of each ring is $R c$, equal to the communication radius of each node. $h$ represents the number of rings. The identification of rings is from 0 to $h-1$. The ring near the BS is the first ring. Initially, each node belonging to first ring $(\mathrm{k}=0)$ starts relay node competition which duration is $T$.

As well as EADC [20], each candidate relay node $\mathrm{r}_{\mathrm{i}}$ has a competition range $R 1$. Only one final relay node is allowed in each competition range.

Each tentative relay node broadcasts a CompeteRelayMsg which contains its residual energy. It maintains a set $S_{\text {relay }}$ of its adjacent tentative relay nodes.

Once the $S_{\text {relay }}$ is created, each candidate makes a decision. If the node $r_{i}$ has the largest residual energy, it is elected as relay node and broadcasts a FinalRelayMsg message. Otherwise, it is out of the competition.

Simultaneously, when the duration $T$ is expired, only node belonging to ring $(k+1)$ receives FinalRelayMsg can start relay node competition. The process will be repeated for all rings. This process guarantees the connectivity of the relays.

\subsection{CH selection and clustering}

This stage is divided into two phases: $\mathrm{CH}$ competition phase and cluster formation phase.

\subsubsection{CH competition phase}

Each node received FinalRelayMsg and it is not chosen as a relay alters its state to a "Tentative". Consequently, the connectivity of cluster heads is achieved. The $\mathrm{CH}$ selection is based on the residual energy of candidate $\mathrm{CH}$ and its density. The role of cluster head is periodically rotated among the nodes to distribute the energy consumption among nodes.

$$
\text { State }\left(s_{i}\right)=\left\{\begin{array}{c}
\text { "Tentative" if Connect }\left(s_{i}\right)=\text { True and } s_{i} \notin R(r) \\
\text { "Member" Otherwise }
\end{array}\right.
$$

Where $\mathrm{R}(\mathrm{r})$ is the set of elected relays.

Connect $\left(s_{i}\right)=1$. The number 1 indicates that the sensor node $s_{i}$ is connected and 0 if otherwise. A node is said connected if $\exists r_{j} \in R(r), d\left(s_{i}, r_{j}\right) \leq R c$

As well as EADC [20], each candidate $\mathrm{CH} \mathrm{s}_{\mathrm{i}}$ has a competition range R2. Only one final $\mathrm{CH}$ is permitted in each competition range.

Each tentative $\mathrm{CH}$ broadcasts a CompeteHeadMsg which contains its residual energy and its density. It maintains a set $\mathrm{S}_{\mathrm{CH}}$ of its adjacent tentative $\mathrm{CH}$.

Once the set $\mathrm{S}_{\mathrm{CH}}$ has finished, each candidate $\mathrm{CH}$ makes a decision: If the node $\mathrm{s}_{\mathrm{i}}$ has the highest weight among all candidates in its $\mathrm{S}_{\mathrm{CH}}$, it is selected as $\mathrm{CH}$ and broadcasts a FinalHeadMsg message. Otherwise, it gives up the competition. For the tentative $\mathrm{CH} \mathrm{j}$, the weight is computed by the following equation:

$$
\text { Weight }_{j}=\operatorname{alpha} \times \frac{E_{\text {Res }}^{j}}{E_{\max }}+(1-\operatorname{alpha}) \times \frac{\operatorname{density}(j)}{N}
$$

\subsubsection{Cluster formation phase}

After selection of $\mathrm{CH}$, each $\mathrm{CH}$ will advertise a Join-Request (JREQ) message to all its neighbor nodes for cluster formation. Each non- $\mathrm{CH}$ node selects a $\mathrm{CH}$ based on its residual energy and distance to eligible CH. Eq. (4) shows this relation.

$$
\operatorname{Choose}\left(s_{i}, C H_{j}\right)=a \times \frac{1}{d\left(s_{i}, C H_{j}\right)}+(1-a) \times \frac{E_{\text {res }}^{C H}}{E_{\max }}
$$

Where $\alpha$ is the weighted factor, $E_{\text {res }}^{C H j}$ is the residual energy of $\mathrm{CHj}$ and $\mathrm{d}\left(\mathrm{s}_{\mathrm{i}}, \mathrm{CH}_{\mathrm{j}}\right)$ is the distance between the member node's $\mathrm{s}_{\mathrm{i}}$ and the eligible $\mathrm{CH}_{\mathrm{j}}$. Based on this formula, each node is attached to the $\mathrm{CH}$ with more residual energy and less distance. This is aimed at saving more energy at every $\mathrm{CH}$ and spreading load between them. 
A Join-Acknowledgement (J-ACK) message will be sent from each non-CH node to its $\mathrm{CH}$ node to become a cluster member. After receiving J-ACK messages, each $\mathrm{CH}$ must maintain a cluster member table. It then creates a TDMA schedule for each cluster member node.

\subsection{Routing tree construction}

The objective of our algorithm is to find out an optimal route from each $\mathrm{CH}$ to the $\mathrm{BS}$ which has comparably lower overall energy consumption with more workload distribution.

The following terminologies will be used in the proposed algorithm:

1. The set of relay is denoted by $R=\left\{r_{1}, r_{2}, \ldots, r_{K}\right\}$ and $r_{K+1}$ indicates the BS.

2. The set of $\mathrm{CH}$ is denoted by $\mathrm{CH}=\left\{\mathrm{CH}_{1}, \mathrm{CH}_{2}, \ldots, \mathrm{CH}_{\mathrm{L}}\right\}$.

3. Sensor node $r_{i}$ is deployed at point $\left(x_{i}, y_{i}\right)$. For any sensor node $r_{j}$ deployed at $\left(x_{j}, y_{j}\right)$, we denote the Euclidean distance between $r_{i}$ and $r_{j}$ as $d\left(r_{i}, r_{j}\right)$.

$$
d\left(r_{i}, r_{j}\right)=\sqrt{\left(x_{i}-x_{j}\right)^{2}+\left(y_{i}-y_{j}\right)^{2}}
$$

In the proposed protocol, nodes with higher residual energy will take more forwarding tasks in order to prolong the network lifetime.

To construct a routing tree, each current relay node makes a decision to select the next hop node. In order to balance load among relay nodes for inter-network communications, a relay node which has smaller number of $\mathrm{CH}$ members is more appropriate to be chosen as a relay node. Besides, relay nodes which have residual energy are better choices for taking the responsibility of relay in inter-cluster communications. Selecting an upper relay node with more residual energy leads to later death of sensor nodes.

Each relay node $\left(r_{i} \mid r_{i} \in\{R U C H\}\right)$ pick out the neighbor relay node $r_{j}\left(r_{j} \in R\right)$. The node with larger residual energy, smaller number of relay members, smaller link cost and no further away from the BS will be selected as next hop. Here, we give the formula of the indicator "Cost" when relay node $r_{i}$ chooses relay node $r_{j}$ as its next hop. The node that has the minimum value of Cost $_{\mathrm{ij}}$ will be selected as the next. The cost function is defined by equation 6 :

$$
\begin{aligned}
& \operatorname{Cost}_{i j}=\text { beta } \times \frac{\operatorname{Erelay}\left(r_{i}, r_{j}\right)}{\text { Relay }_{\max }}+(1-\text { beta }) \times\left(\frac{E_{\text {max }}-E_{r e s}^{r j}}{E \max }+\frac{r_{j} . \text { members }}{N}\right) \\
& \operatorname{Relay}_{\text {max }}=\max \left\{\operatorname{Erelay}\left(r_{i}, r_{j}\right)\right\}, r_{j} \in F
\end{aligned}
$$

Here $E_{\text {res }}$ represents the residual energy of cluster head $r j E_{\max }$ indicates the initial energy of nodes in the network. $r_{j}$.members represents the number of relay members of $r_{j}$ and beta is a real value uniformly distributed in $[0,1] . F(i)$ defines the neighbor relay node set of $i, F(i)$ can be written as follow:

$$
\begin{gathered}
F(i)=\{j \mid d(i, B S)>d(j, B S), j \in R\} \\
E_{\text {relay }}\left(r_{i}, r_{j}\right)=d^{2}\left(r_{i}, r_{j}\right)+d^{2}\left(r_{i}, B S\right)
\end{gathered}
$$

We can see from the formula, the relay node with higher residual, smaller link cost and fewer relay members will have a small "Cost". Each relay selects the neighbor relay node with the smallest "Cost" as the next hop. Thus, the energy consumption is well-balanced among relay nodes and the transmission cost in the data forwarding process is minimized. The following pseudo-code gives the details of this routing algorithm. 


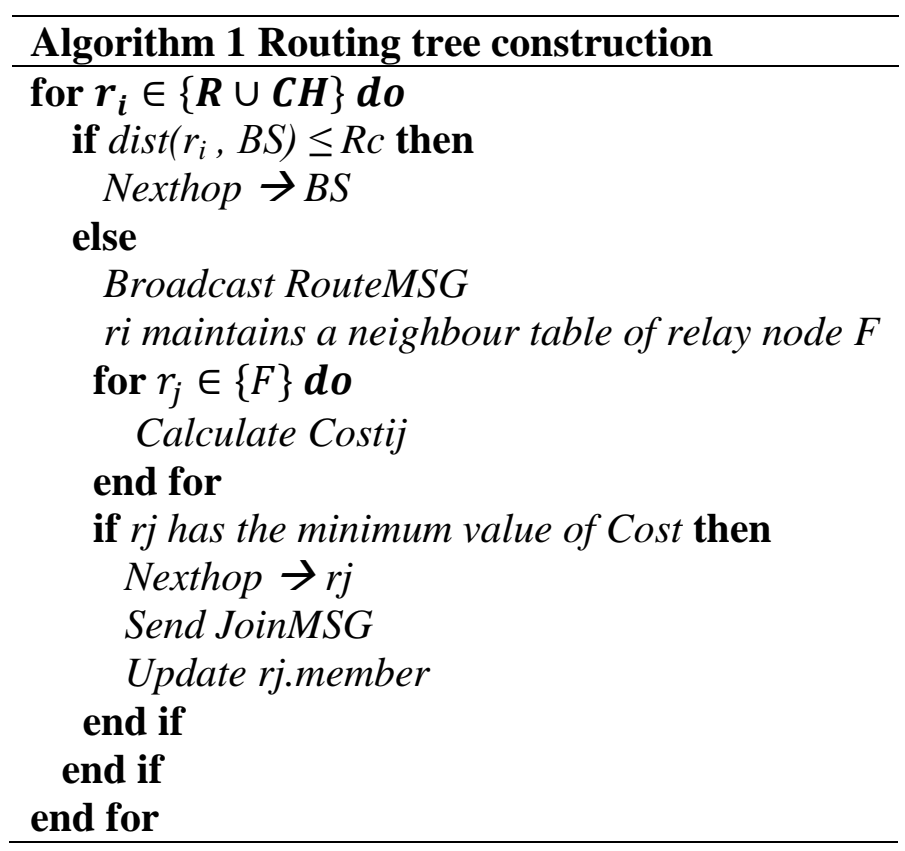

\subsection{Data transmission}

The data transmission is divided into three steps. First is intra-cluster where each cluster member send data to its $\mathrm{CH}$. the second is to send the aggregated data to the relay nodes. The third is to transmit the data to BS. In data forwarding process, relay nodes work together to send their data to the BS.

Every cluster member transmits the data to $\mathrm{CH}$ according the TDMA schedule. When all data are received, each $\mathrm{CH}$ decides whether it will send the message to base station or not.

The node can sense and send incorrect values to its $\mathrm{CH}$. To enhance the accuracy of data and decrease the number of erroneous messages transmitted to the $\mathrm{BS}$, a consensus is required to generate agreement about sensor' measured data. A node will only detect an event within its sensing range when the measured data exceeds a predefined threshold $S$ (Gain fault) and the data must not exceed a certain limit (out-bound fault). The $\mathrm{CH}$ will trigger an event when a threshold $(T h)$ has reached [21].

$$
\frac{n}{S} \times 100 \geq T h
$$

Where $n$ is the number of nodes that detect an event and $S$ denotes the number of nodes which participate in this quorum.

Once transfer decision is made, $\mathrm{CH}$ forward data to the $\mathrm{BS}$ along constructed routing tree.

\section{SIMULATIONS AND RESULTS}

\subsection{Experiment settings and metrics}

To evaluate the proposed protocol, the same radio energy model with [24] is adopted. To transmit a $b$-bit message over a distance $d$, the energy consumed is calculated using following equations:

$$
\begin{gathered}
E^{t x}(b, d)=E_{e}^{t x}(b)+E_{a}^{t x}(b, d)=b \times E_{e}+E_{a}(b, d) \\
E_{a}(b, d)=\left\{\begin{array}{l}
b \times E_{\text {friss-amp }} \times d^{2} \text { if } d \leq d_{c} \\
b \times E_{t w o-r a y-a m p} \times d^{4} \text { if } d>d_{c}
\end{array}\right.
\end{gathered}
$$

And the energy for receiving a b-bit message is estimated through formula 13: 


$$
E^{r x}(b)=E_{e}^{r x}(b)=b \times E_{e}
$$

In this experiment, $\mathrm{N}$ sensor nodes are randomly deployed in a sensing field of $200 \mathrm{~m}$ x $200 \mathrm{~m}$ square area. The simulation parameters are given in Table 1.

Table 1. Simulation Parameters.

\begin{tabular}{|c|c|}
\hline Parameters & Values \\
\hline Network Field & $200 \times 200$ \\
\hline BS location & $(200,100)$ \\
\hline $\mathrm{N}$ & 400 \\
\hline $\mathrm{E}_{\mathrm{e}}$ & $50 \mathrm{~nJ} / \mathrm{bit}$ \\
\hline $\mathrm{E}_{\text {friss-amp }}$ & $10 \mathrm{pJ} / \mathrm{bit} / \mathrm{m}^{2}$ \\
\hline $\mathrm{E}_{\text {two-ray-amp }}$ & $0.0013 \mathrm{pJ} / \mathrm{Bit} / \mathrm{m}^{4}$ \\
\hline Dc & 87 \\
\hline Data packet size & 50 Bytes \\
\hline Initial Energy of sensor $\mathrm{E}_{0}$ & $0.5 \mathrm{~J}$ \\
\hline
\end{tabular}

In this paper, the performance our protocol will be evaluated for the following metrics: First Node Dies (FND) and Half of the Nodes Alive (HNA). If a node's residual energy is less than the value required for the transmission task, it is considered "dead".

\subsection{AEEC without fault detection}

AEEC is compared with an energy-aware clustering algorithm (EADC) and Energy-Efficient Unequal Clustering (EEUC).

We set R1 from 40 to 80 and R2 from 40 to 80 . Then, we run AEEC. The variation of network lifetime along with the variation R1 and R2 is shown in Figure 2.

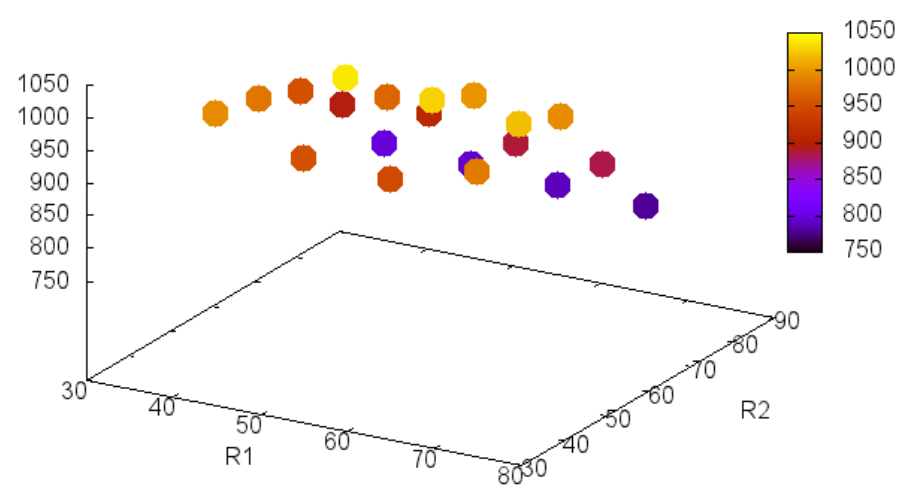

Figure 2. Network lifetime under R1 and R2 variation.

Results show that network lifetime reach the optimal point when $\mathrm{R} 1=50$ and $\mathrm{R} 2=50$.

The proposed algorithm could achieve well distribution of $\mathrm{CHs}$ and relay node in the whole network. The well distribution of cluster heads and relay nodes over the sensing field is accomplished using the competition radius mechanism.

We set alpha from 0.0 to 1.0 , beta from 0.0 to $1.0, \mathrm{R} 1=50$ and $\mathrm{R} 2=50$. Then, we run AEEC. The variation of network lifetime along with the variation of Alpha and Beta is shown in Figure 3. Results show that network lifetime reach the optimal point when Alpha $=0.2$ and Beta $=0.6$. 


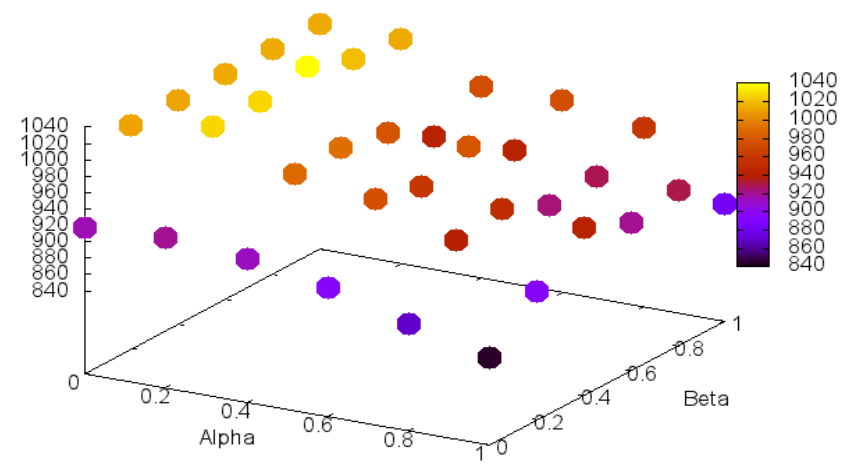

Figure 3. Network lifetime under Alpha and Beta variation.

Simulations above that compare AEEC with an energy-aware clustering algorithm (EADC) and Energy-Efficient Unequal Clustering (EEUC) prove that we can reach the optimal point when $\mathrm{R} 1=50, \mathrm{R} 2=50$ Alpha $=0.2$ and Beta $=0.6$.

The selection of cluster heads is based on remaining energy of nodes and distance. Therefore, the intra-cluster communication cost will be minimized and the energy consumption among $\mathrm{CH}$ will be more balanced. In addition, to reduce imbalance of energy consumption among relay nodes, a trade-off should be made between the three criteria of link cost, residual energy and relay members.

Table 2 gives an overview of the percentage of $\mathrm{CHs}$ and the average of energy consumed by $\mathrm{CH}$ in 200 randomly selected rounds for EADC, EEUC and the proposed AEEC method.

Table 2 Percentage of $\mathrm{CH}$ and energy consumed

\begin{tabular}{|c|c|c|c|c|c|c|c|}
\hline \multicolumn{2}{|c|}{ EADC } & \multicolumn{2}{c|}{ EEUC } & \multicolumn{4}{c|}{ AEEC } \\
\hline $\mathrm{CH}(\%)$ & Energy (J) & $\mathrm{CH}(\%)$ & Energy $(\mathrm{J})$ & $\mathrm{CH}(\%)$ & Energy (J) & Relay (\%) & Energy (J) \\
\hline 4 & 0.104 & 3 & 0.093 & 3 & 0.084 & 2 & 0.008 \\
\hline
\end{tabular}

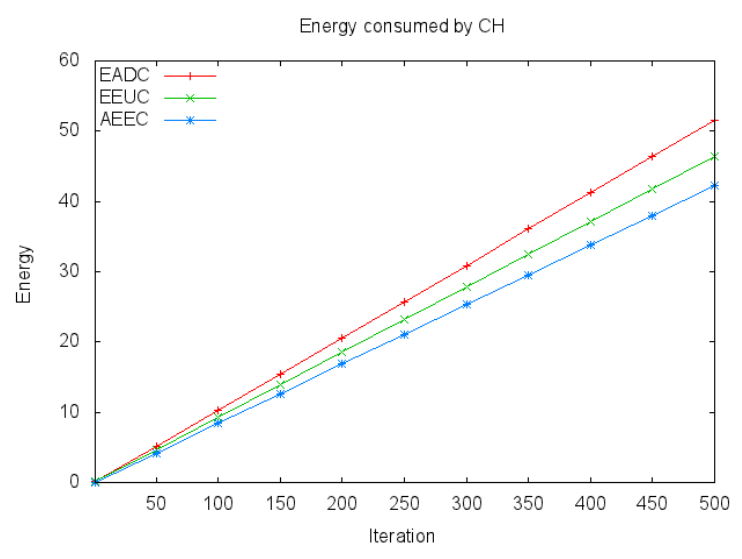

Figure 4 Variance of energy consumed by $\mathrm{CH}$.

As shown in Table 2 and Figure 4, the energy consumption by $\mathrm{CH}$ of the proposed protocol is less than that of EADC and EEUC. In EADC and EEUC, $\mathrm{CH}$ carries some extra workload contributed by their cluster members as they receive the sensed data from their cluster members, aggregate them and communicate it to the BS. Thus, $\mathrm{CH}$ consumes more energy during message transmission to BS. To avoid this problem, the proposed protocol selects a collection of powerful relay nodes from the normal sensor nodes and a routing tree would be constructed on elected relay nodes set to forward data to the BS. In the proposed protocol, the energy 
consumption among nodes is more balanced. Thus, AEEC avoids premature death of relay $\mathrm{CH}$ close to the BS.

Figure 5 illustrates the comparison between EADC, EEUC and AEEC in term of network lifetime. As shown in Figure 5, FND is reached later under AEEC (1040 rounds) than under EADC (795 rounds) and under EEUC (972 rounds). Moreover, HND is reached later under AEEC (1067 rounds) than under EADC (1028 rounds) and under EEUC (1057 rounds).

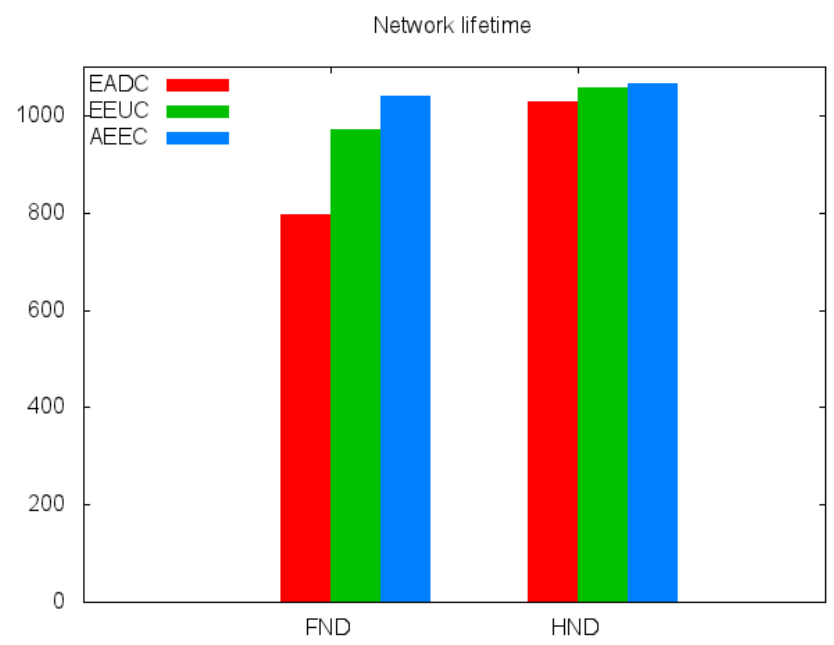

Figure 5 Network lifetime

Figure 6 illustrates the number of nodes still alive in each round. As shown in Figures 5 and 6 , in the proposed protocol, the energy consumption is more balanced. Therefore, AEEC gives better performances than EADC and EEUC in extending the network lifetime. AEEC overcomes the imbalance energy consumption problem by distributing tasks among nodes.

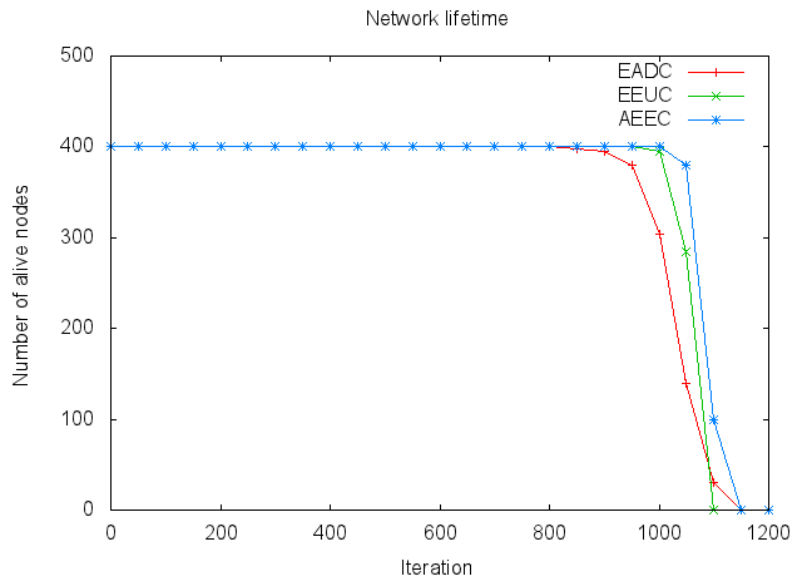

Figure 6 Network lifetime

According to the simulation above, we can conclude that AEEC can avoid the premature death of nodes closer to the BS and prolong the network lifetime significantly.

\subsection{AEEC with fault detection}

In this network, $N$ sensors are deployed to continuously detect events. Events are randomly distributed throughout the entire network. It is assumed that every node will continuously sense and send the data to its $\mathrm{CH}$. Next, the decision function will be executed at cluster heads to detect faulty data. The $\mathrm{CH}$ does not send any data unless there is a consensus about it. Our approach is evaluated where faults are essentially gained and out of bounds fault. 
We introduced a set of random faults for each iteration. We executed the proposed algorithm by varying the rates of faults from 5\% to $25 \%$ and Threshold (Th) from 0.51to 0.67. As shown in Figure 7, when the probability of faults increases, the rate of detection decreases and there will be more risks of faulty data forwarded to a base station.

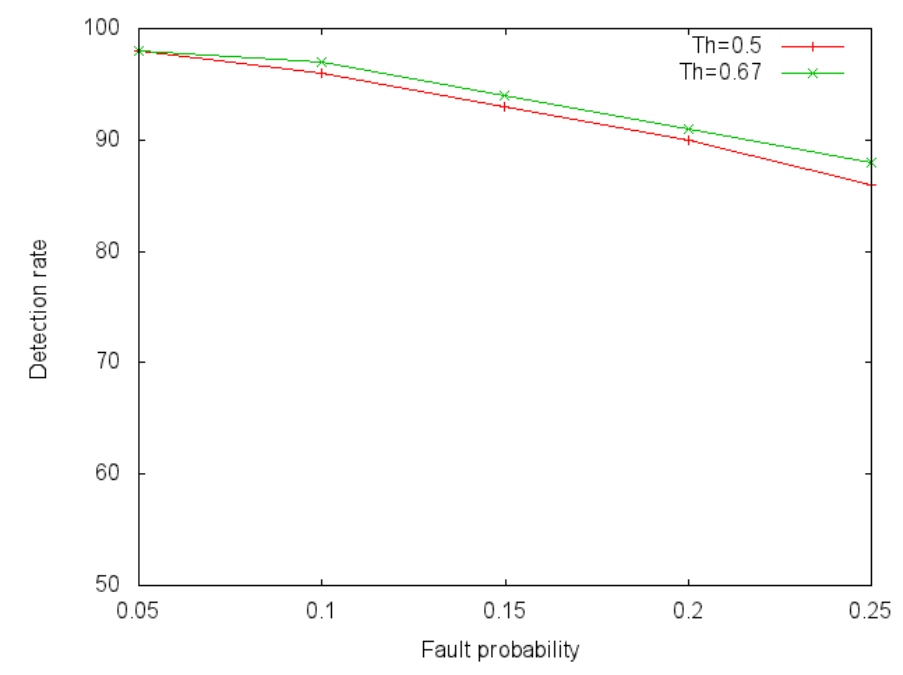

Figure 7 Detection rate

In this experiment, we set the probability of fault to $5 \%$ and $T h$ to 0.51 . Figure 8 shows the comparison between AEEC and I-AEEC with fault detection in term of number of erroneous messages sent to base station. As shown in this figure, the number of transmissions is reduced and thus incorrect and costly actions are minimized. As a result, the consumed energy is minimized; therefore the network lifetime will be enhanced.

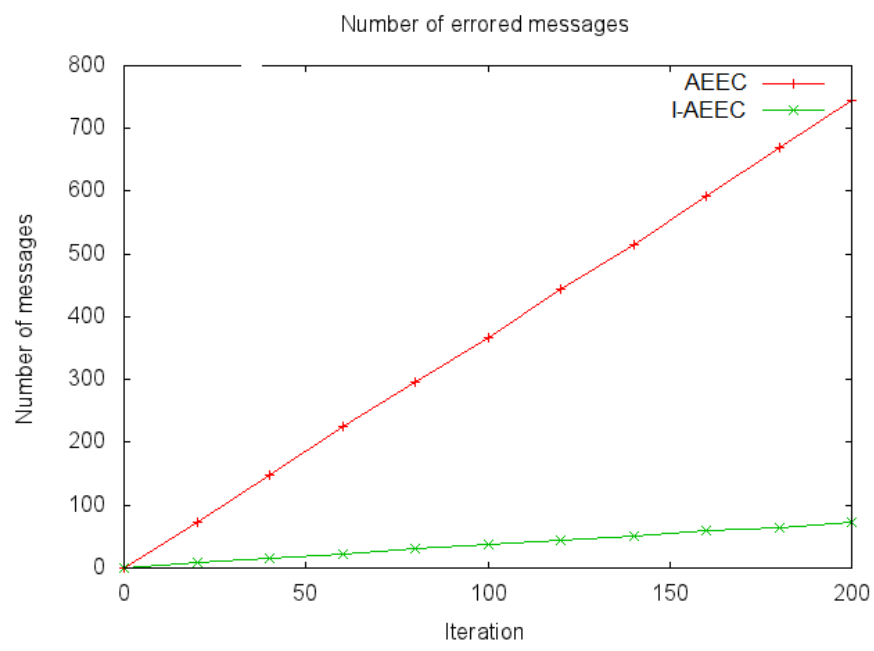

Figure 8 Number of erroneous messages sent to BS

Based on simulations tested above, in data transmission, a consensus is required to generate agreement about sensor measurements. Consequently, our algorithm (I-AEEC) minimizes the number of erroneous messages transmitted. Thus, data accuracy is enhanced.

\section{CONCLUSION}

In this paper, an energy-efficient protocol called AEEC is presented. The proposed protocol improves the energy efficiency and the distribution of $\mathrm{CH}$ nodes across the network. In order to avoid the long-haul communication links and imbalanced energy consumption between $\mathrm{CH}$, the 
proposed protocol selects a collection of powerful relay nodes from the normal sensor nodes and a routing tree would be constructed on elected relay nodes set to forward data to the BS.

To minimize negative alerts and incorrect actions, an approach of fault detection based on a consensus is developed: a threshold of credibility tries to minimize the number of erroneous transmissions and therefore, considerable energy conservation is attained.

The experimental results show that the proposed algorithm performs better than the associated algorithm in terms of overall energy consumption, network lifetime and data accuracy.

Based on relevant results obtained during this work, for the coming works, we will experimentally evaluate and verify in real deployment scenarios.

\section{REFERENCES}

[1] N. Ramachandran and V. Perumal, Delay-aware heterogeneous cluster-based data acquisition in internet of things," Computers \& Electrical Engineering, vol. 65, pp. $44\{58,2018$.

[2] A. Mehmood, J. Lloret, and S. Sendra, \A secure and low-energy zone-based wireless sensor networks routing protocol for pollution monitoring," Wireless Communications and Mobile Computing, vol. 16, no. 17, pp. 2869\{2883, 2016.

[3] B. Mo, W. Dong, C. Chen, J. Bu, and Q. Wang, An efficient differencing algorithm for reprogramming wireless sensor networks," Ad Hoc \& Sensor Wireless Networks, vol. 21, no. 34, pp. 201-218, 2014.

[4] G. Abdul-Salaam, A. H. Abdullah, M. H. Anisi, A. Gani, and A. Alelaiwi, A comparative analysis of energy conservation approaches in hybrid wireless sensor networks data collection protocols," Telecommunication Systems, vol. 61, no. 1, pp. 159-179, 2016.

[5] I. A. J. P. Ahmed Patel, Samaher Al-Janabi, A novel methodology towards a trusted environment in mashup web applications," computers and security, vol. 49, no. 1, pp. 107-122, 2015.

[6] M. S. S. S. Samaher Al-Janabi, Ibrahim Al-Shourbaji, Survey of main challenges (security and privacy) in wireless body area networks for healthcare applications," Egyptian Informatics Journal, vol. 18, no. 1, pp. 113-122, 2017.

[7] J. Huang, Q. Duan, C. Xing, and H. Wang, Topology control for building a large-scale and energy-efficient internet of things," IEEE Wireless Commun., vol. 24, no. 1, pp. 67-73, 2017.

[8] Z. Sheng, D. Tian, and V. C. M. Leung, Toward an energy and resource efficient internet of things: A design principle combining computation, communications, and protocols," IEEE Communications Magazine, vol. 56, no. 7, pp. 89-95, 2018.

[9] L. Liang, L. Xu, B. Cao, and Y. Jia, A cluster-based congestion-mitigating access scheme for massive M2M communications in internet of things," IEEE Internet of Things Journal, vol. 5, no. 3, pp. 2200-2211, 2018.

[10] E. P. K. Gilbert, B. Kaliaperumal, E. B. Rajsingh, and M. Lydia, Trust based data prediction, aggregation and reconstruction using compressed sensing for clustered wireless sensor networks," Computers \& Electrical Engineering, vol. 72, pp. 894-909, 2018.

[11] H. Zhong, L. Shao, J. Cui, and Y. Xu, An efficient and secure recoverable data aggregation scheme for heterogeneous wireless sensor networks," J. Parallel Distrib. Comput., vol. 111, pp. 1-12, 2018.

[12] C. Li, J. Bai, J. Gu, Y. Xin, and Y. Luo, Clustering routing based on mixed integer programming for heterogeneous wireless sensor networks," Ad Hoc Networks, vol. 72, pp. 8190, 2018.

[13] S. Arjunan and P. Sujatha, Lifetime maximization of wireless sensor network using fuzzy based unequal clustering and ACO based routing hybrid protocol," Appl. Intell., vol. 48, no. 8, pp. 2229-2246, 2018.

[14] G. P. Gupta and S. Jha, Untegrated clustering and routing protocol for wireless sensor networks using cuckoo and harmony search based metaheuristic techniques," Eng. Appl of AI, vol. 68, pp. 101-109, 2018.

[15] A. S. Rostami, M. Badkoobe, F. Mohanna, H. Keshavarz, A. A. R. Hosseinabadi, and A. K. Sangaiah, Survey on clustering in heterogeneous and homogeneous wireless sensor networks," The Journal of Supercomputing, vol. 74, no. 1, pp. 277\{323, 2018.

[16] K. A. Darabkh, M. Z. El-Yabroudi, and A. H. El-Mousa, BPA-CRP: A balanced power-aware clustering and routing protocol for wireless sensor networks," Ad Hoc Networks, vol. 82, pp. 155-171, 2019. 
[17] P. Kuila and P. K. Jana, IEnergy efficient clustering and routing algorithms for wireless sensor networks: Particle swarm optimization approach," Engineering Applications of Artificial Intelligence, vol. 33, pp. 127-140, 2014.

[18] M. Sabet and H. R. Naji, \An energy efficient multi-level route-aware clustering algorithm for wireless sensor networks: A self-organized approach," Computers \& Electrical Engineering, vol. 56, pp. 399-417, 2016.

[19] T. Muhammed and R. A. Shaikh, An analysis of fault detection strategies in wireless sensor networks," J. Network and Computer Applications, vol. 78, pp. 267-287, 2017.

[20] J. Yua, Y. Qia, G. Wangb, and X. Gua, A cluster-based routing protocol for wireless sensor networks with nonuniform node distribution," International Journal of Electronics and Communications (AEU), vol. 66, pp. 54-61, 2012.

[21] S. Chelbi, C. Duvallet, M. Abdouli, and R. Bouaziz, Event-driven wireless sensor networks based on consensus," in 13th IEEE/ACS International Conference of Computer Systems and Applications, AICCSA 2016, Agadir, Morocco, 2016, 2016, pp. 1-6.

[22] G. Chen, C. Li, M. Ye, and J.Wu, An unequal cluster-based routing protocol in wireless sensor networks," Wireless Networks, vol. 15, no. 2, pp. 193-207, 2009.

[23] H. B. P. C. Srinivasa Rao, Prasanta K. Jana, A particle swarm optimization based energy efficient cluster head selection algorithm for wireless sensor networks," Wireless Networks, vol. 23, no. 7, pp. 2005-2020, 2017.

[24] W. B. Heinzelman, A. P. Chandrakasan, and H. Balakrishnan, An application-specific protocol architecture for wireless microsensor networks," IEEE Transactions on Wireless Communications, vol. 1, no. 4, pp. 660-670, 2002. 